\title{
Nuclear Structure in Nonmesonic Weak Decay of Hypernuclei
}

\author{
F. Krmpotić \\ Departamento de Física, Universidad Nacional de La Plata, \\ C. C. 67, 1900 La Plata, Argentina \\ and D. Tadić \\ Physics Department, University of Zagreb, 10000 Zagreb, Croatia
}

Received on 30 October, 2002

\begin{abstract}
A general shell model formalism for the nonmesonic weak decay of the hypernuclei has been developed. It involves a partial wave expansion of the emitted nucleon waves, preserves naturally the antisymmetrization between the escaping particles and the residual core, and contains as a particular case the weak $\Lambda$-core coupling formalism. The hypernuclei are grouped having in view their $A-1$ cores, that is in those with even-even, evenodd and odd-odd cores. It is shown that in all three cases the nuclear structure manifests itself basically through Pauli Principle, and very simple expressions are derived for the neutron and proton induced decays rates, $\Gamma_{n}$ and $\Gamma_{p}$, which does not involve the spectroscopic factors. For the strangeness-changing weak $\Lambda N \rightarrow N N$ transition potential we use the One-Meson-Exchange Model (OMEM), which comprises the exchange of the complete pseudoscalar and vector meson octets $\left(\pi, \eta, K, \rho, \omega, K^{*}\right)$. We evaluate ${ }^{3} \mathrm{H},{ }^{4} \mathrm{H},{ }_{\Lambda}^{4} \mathrm{He},{ }_{\Lambda}^{5} \mathrm{He},{ }^{11} \mathrm{~B}$, ${ }_{\Lambda}^{12} \mathrm{C},{ }_{\Lambda}^{16} \mathrm{O},{ }_{\Lambda}^{17} \mathrm{O}$, and ${ }_{\Lambda}^{28} \mathrm{Si}$ hypernuclei, with commonly used parametrization for the OMEM, and compare the results with the available experimental information. The calculated rates $\Gamma_{N M}=\Gamma_{n}+\Gamma_{p}$ are consistent with the data, but the measurements of $\Gamma_{n / p}=\Gamma_{n} / \Gamma_{p}$ are not well accounted for by the theory. It is suggested that, unless additional degrees of freedom are incorporated, the OMEM parameters should be radically modified.
\end{abstract}

\section{Introduction}

Hypernuclear physics adds another flavor (strangeness) to the traditional nuclear physics, and its goal is to study the behavior of hyperons $(\Lambda, \Sigma, \Xi, \Omega)$ in the nuclear environments, which are now bound system of neutrons, protons and one or more hyperons. Interesting strange nuclei with strangeness $S=-1$ are the $\Lambda$ hypernuclei, in which a $\Lambda$ hyperon, having a mass of $1116 \mathrm{MeV}$ and zero charge and isospin, replaces one of the nucleons. Same as the free $\Lambda$ hyperon, they are mostly produced via the strong interactions, i.e., in the reaction processes $\pi^{+} n \rightarrow \Lambda K^{+}, K^{-} n \rightarrow \pi^{-} \Lambda$ and $K^{-} p \rightarrow \pi^{0} \Lambda$, by making use of the pion $(\pi)$ and kaon $(K)$ beams. They also basically decay through the weak interactions, as the free $\Lambda$ does. Yet, as it is well known and explained below, there are some very important differences in the corresponding decaying modes.

First, it should be remembered that the free $\Lambda$ hyperon decays nearly $100 \%$ of the time by the $\Lambda \rightarrow N \pi$ weakmesonic mode (Fig. 1):

$$
\Lambda \rightarrow \begin{cases}p+\pi^{-} & (64.1 \%) \\ n+\pi^{0} & (35.7 \%)\end{cases}
$$

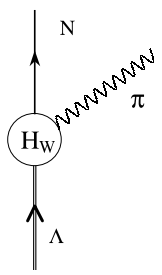

Figure 1. The mesonic (nonleptonic) decay vertex $\mathcal{H}_{\Lambda N \pi}^{W}$.

with the total transition rate $\Gamma_{\pi^{-}}^{0}+\Gamma_{\pi^{0}}^{0}=\Gamma^{0}=2.50 \cdot 10^{-6}$ $\mathrm{eV}$ (which corresponds to the lifetime $\tau^{0}=2.63 \cdot 10^{-10}$ $\mathrm{sec})$. For the decay at rest the energy-momentum conservation implies

$$
+M_{\Lambda}=M_{N}+\frac{p_{N}^{2}}{2 M_{N}}+\sqrt{p_{\pi}^{2}+m_{\pi}^{2}} ; \quad p_{N} \equiv p_{\pi} .
$$

Therefore the energy released is

$$
Q_{0}=M_{\Lambda}-M_{N}-m_{\pi} \cong 37 \mathrm{MeV},
$$

and the kinetic energies and momenta in the final state are:

$$
\begin{aligned}
T_{N} & =\frac{\left(M_{\Lambda}-M_{N}\right)^{2}+m_{\pi}^{2}}{2 M_{\Lambda}} \cong 5 \mathrm{MeV} \\
T_{\pi} & =Q_{0}-T_{N} \cong 32 \mathrm{MeV}, \\
p_{N} & \equiv p_{\pi}=\sqrt{\left(T_{N}+M_{N}\right)^{2}-M_{N}^{2}} \cong 100 \mathrm{MeV} / c .
\end{aligned}
$$


It is clear that the isospin is changed by $\Delta T=1 / 2$ and $3 / 2$

decay. More, as and its projection by $\Delta M_{T}=-1 / 2$ in the free $\Lambda \rightarrow N \pi$

- $\Lambda \rightarrow n+\pi^{0}$ :

$$
|n\rangle\left|\pi^{0}\right\rangle \equiv|1 / 2,-1 / 2\rangle|10\rangle=\sqrt{\frac{1}{3}}|1 / 2,1 ; 1 / 2,-1 / 2\rangle+\sqrt{\frac{2}{3}}|1 / 2,1 ; 3 / 2,-1 / 2\rangle
$$

- $\Lambda \rightarrow p+\pi^{-}$:

$$
|p\rangle\left|\pi^{-}\right\rangle \equiv|1 / 2,1 / 2,\rangle|1,-1\rangle=-\sqrt{\frac{2}{3}}|1 / 2,1 ; 1 / 2,-1 / 2\rangle+\sqrt{\frac{1}{3}}|1 / 2,1 ; 3 / 2,-1 / 2\rangle,
$$

one sees that the above experimental data can be accounted for fairly well by neglecting the $|1 / 2,1 ; 3 / 2,-1 / 2\rangle$ components in these relations $\left(\Delta T=1 / 2\right.$ rule). In fact, one gets $\Gamma_{\pi^{-}} / \Gamma_{\pi^{0}}=2$, while the experimental result is $64.1 / 35.7=1.80$.

Assuming the $\Delta T=1 / 2$ rule, the phenomelogical weak Hamiltonian for the process depicted in Fig. 1 can be expressed as:

$$
\mathcal{H}_{\Lambda N \pi}^{W}=-i G_{F} m_{\pi}^{2} \bar{\psi}_{N}\left(A_{\pi}+B_{\pi} \gamma_{5}\right) \phi_{\pi} \cdot \boldsymbol{\tau} \psi_{\Lambda}\left(\begin{array}{l}
0 \\
1
\end{array}\right)
$$

where $G_{F} m_{\pi}^{2}=2.21 \times 10^{-7}$ is the weak coupling constant. The empirical constants $A_{\pi}=1.05$ and $B_{\pi}=-7.15$, adjusted to the observables of the free $\Lambda$ decay, determine the strengths of parity violating and parity conserving amplitudes, respectively. The nucleon, $\Lambda$ and pion fields are given by $\psi_{N}$ and $\psi_{\Lambda}$ and $\phi_{\pi}$, respectively, while the isospin spurion $\left(\begin{array}{l}0 \\ 1\end{array}\right)$ is included in order to enforce the empirical $\Delta T=1 / 2$ rule. (Note that: $\bar{\psi}_{N} \phi_{\pi} \cdot \boldsymbol{\tau} \psi_{\Lambda}=$

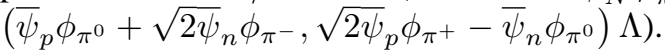

The free $\Lambda$ hyperon weak decay is radically modified in the nuclear environment because the nucleon and the hyperon now move, respectively, in the mean fields $U_{N}$ and $U_{\Lambda}$, which come from the $N N$ and $N \Lambda$ interactions. $U_{N}$ and $U_{\Lambda}$ are characterized by the single particle energies (s.p.e.) $\varepsilon_{N}$ and $\varepsilon_{\Lambda}$ and we have to differentiate between:

1. Mesonic Decay (MD): The basic process is again represented by the graph shown in Fig. 1 and described by the hamiltonian (1). Yet, the energy-momentum conservation is different:

$$
\begin{aligned}
M_{\Lambda} & =M_{N}-\varepsilon_{\Lambda}+\varepsilon_{N}^{\uparrow}+\frac{p_{A}^{2}}{2 M_{A}}+\sqrt{p_{\pi}^{2}+m_{\pi}^{2}} \\
\mathbf{p}_{A} & =-\mathbf{p}_{\pi}
\end{aligned}
$$

where $M_{A}=A M_{N}$ and $\mathbf{p}_{A}$ are, respectively, the mass and the momentum of the whole nucleus; $A$ is the mass number, and $\varepsilon_{N}^{\uparrow}$ are the s.p.e. of the loosely bound states above the Fermi energy $\varepsilon_{N}^{F}$. They are of the order of a few $\mathrm{MeV}$, while $\varepsilon_{\Lambda}$ is the energy of the $0 s_{1 / 2}$ state and goes from $-11.7 \mathrm{MeV}$ for ${ }_{\Lambda}^{13} C$ to $-26.5 \mathrm{MeV}$ for ${ }_{\Lambda}^{208} \mathrm{~Pb}$ [1]. Thus, the corresponding Q-values

$$
Q_{M}=M_{\Lambda}-M_{N}-m_{\pi}+\varepsilon_{\Lambda}-\varepsilon_{N}^{\uparrow},
$$

are significantly smaller than $Q_{0}$, particularly for medium and heavy nuclei. The experimental decay rates $\Gamma_{\pi^{-}}+$ $\Gamma_{\pi^{0}}=\Gamma_{M} \equiv \Gamma_{M}(\Lambda \rightarrow N \pi)$ are of the order of $\Gamma^{0}$ only for nuclei with $A \leq 4$, and they rapidly fall as a function of nuclear mass. For instance, in ${ }_{\Lambda}^{12} C: \Gamma_{\pi^{0}} / \Gamma^{0}=0.217 \pm 0.084$ and $\Gamma_{\pi^{-}} / \Gamma^{0}=0.052_{-0.035}^{+0.063}$. This hindrance effect, as is illustrated in Fig. 2 for the hypernucleus ${ }_{\Lambda}^{17} \mathrm{O}$, is due to Pauli principle. In fact, the population of states that are below the Fermi level, with energies $\varepsilon_{N}^{\downarrow} \leq \varepsilon_{N}^{F}$, is totally blocked by the Pauli principle, while transitions to states that lie above $\varepsilon_{N}^{F}$ are strongly hindered due to the selection rule $\Delta \mathrm{N}=0$, $\mathrm{N}$ being the harmonic oscillator quantum numbers. That is, only a few second forbidden transitions $(\Delta \mathrm{N}=2)$ can occur in the case of ${ }_{\Lambda}^{17} \mathrm{O}$, and it is clear that the degree of forbiddiness increases with $A$.

2. Nonmesonic Decay: New nonmesonic decay (NMD) channels $\Lambda N \rightarrow N N$ become open inside the nucleus, where there are no pions in the final state. The corresponding transition rates can be stimulated either by protons, $\Gamma_{p} \equiv \Gamma(\Lambda p \rightarrow n p)$, or by neutrons, $\Gamma_{n} \equiv \Gamma(\Lambda n \rightarrow n n)$. The energy-momentum conservation and the $\mathrm{Q}$-value are, respectively:

$$
\begin{aligned}
M_{\Lambda} & =M_{N}-\varepsilon_{\Lambda}-\varepsilon_{N}^{\downarrow}+\frac{p_{1}^{2}}{M_{N}}+\frac{p_{2}^{2}}{M_{N}}+\frac{p_{A}^{2}}{A M_{N}} ; \\
\mathbf{p}_{A} & =-\mathbf{p}_{1}-\mathbf{p}_{2},
\end{aligned}
$$




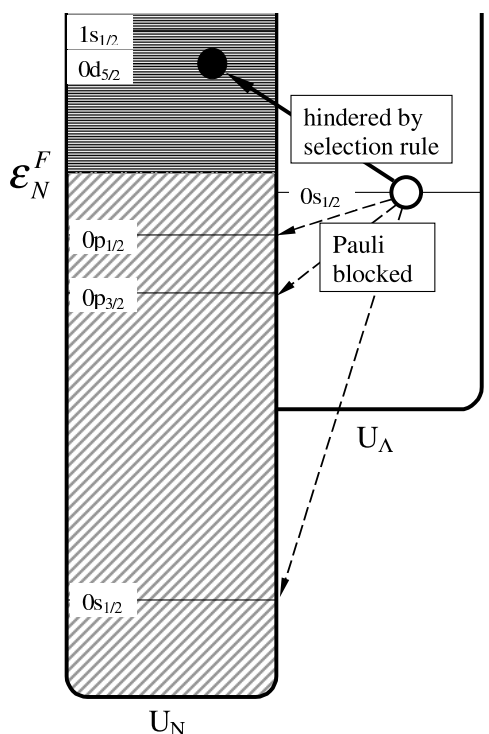

Figure 2. Schematic picture of the hypernuclear mesonic decay in ${ }_{\Lambda}^{17} O$ : The population of occupied states $0 s_{1 / 2}, 0 p_{3 / 2}$ and $0 p_{1 / 2}$, which are below the Fermi level $\varepsilon_{N}^{F}$, is totally blocked by the Pauli principle, while transitions to the weakly bound empty states $0 d_{5 / 2}$ and $1 s_{1 / 2}$, which lie above $\varepsilon_{N}^{F}$, are strongly hindered by the selection rule $\Delta \mathrm{N}=0$.

and

$$
Q_{N M}=M_{\Lambda}-M_{N}+\varepsilon_{\Lambda}+\varepsilon_{N}^{\downarrow},
$$

where $\mathbf{p}_{1}$ and $\mathbf{p}_{2}$ are the momenta of the two outgoing nucleons. As the mean value of $\varepsilon_{N}^{\downarrow}$ is $\sim 30 \mathrm{MeV}$ one gets that $Q_{N M} \sim 120-135 \mathrm{MeV}$, which is basically the kinetic energy of the two particles that are ejected from the hypernucleus. This means that the nonnesonic decay process possesses a large phase space in the continuum, as is outlined in Fig. 3 for the case of the hypernucleus ${ }_{\Lambda}^{17} O$. The theoretical models reproduce fairly well the experimental values of the total width $\Gamma_{N M}=\Gamma_{n}+\Gamma_{p}\left(\Gamma_{N M}^{\exp } \cong \Gamma^{0}\right)$ but the ratio $\Gamma_{n / p} \equiv \Gamma_{n} / \Gamma_{p}\left(0.5 \leq \Gamma_{n / p}^{\exp } \leq 2\right)$ remains a puzzle.

Very often it is assumed that the hypernuclear NMD $\Lambda N \rightarrow N N$ is triggered via the exchange of a virtual meson, and the obvious candidate is the one-pion-exchange (OPE) mechanism, where the strong Hamiltonian

$$
\mathcal{H}_{N N \pi}^{S}=i g_{N N \pi} \bar{\psi}_{N} \gamma_{5} \boldsymbol{\pi} \cdot \boldsymbol{\tau} \psi_{N},
$$

(with $g_{N N \pi}=13.4$ ) accompanies the weak Hamiltonian (1). Following the pioneering investigations of Adams [12] several calculations have been done within this coupling scheme yielding: $\Gamma_{N M}^{(\mathrm{OPE})} \cong \Gamma^{0}$ and $\Gamma_{n / p}^{(\mathrm{OPE})} \cong 0.1-0.2$ [14-39]. The importance of the $\rho$ meson in the weak decay mechanism was first discussed by McKellar and Gibson [13], and the present-day consensus is, however, that the effect of the $\rho$-meson on both $\Gamma_{N M}$ and $\Gamma_{n / p}$ is small [21, 23, $24,26,38]$. The full one meson-exchange model (OMEM), which encompasses all pseudoscalar mesons $(\pi, \eta, K)$ and all vector mesons $\left(\rho, \omega, K^{*}\right)$, has been also considered by several authors $[23,24,33,34,35,36,38]$. From these works we have learned that, although the $K$ meson contribution significantly increases the ratio $\Gamma_{n / p}$, the OMEM is unable to account for the corresponding experimental values.

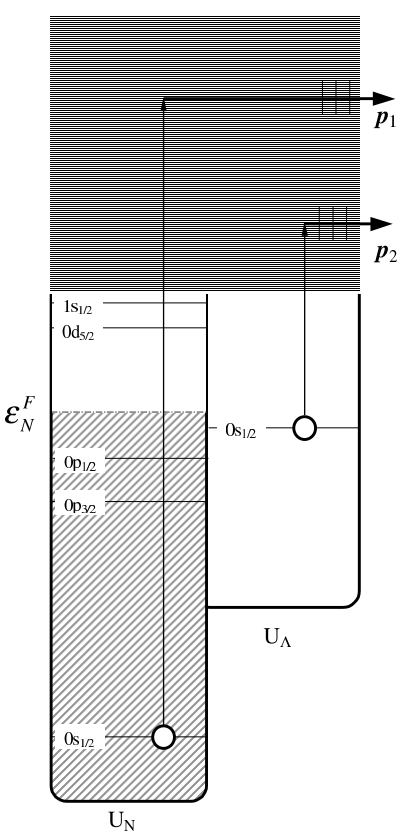

Figure 3. Schematic picture of the hypernuclear nonmesonic decay in ${ }_{\Lambda}^{17} O$ : The hyperon $\Lambda$ and one of the nucleons from the occupied states $0 s_{1 / 2}, 0 p_{3 / 2}$ and $0 p_{1 / 2}$ are expelled into the large phase space in the continuum, becoming free nucleons with momenta $\mathbf{p}_{1}$ and $\mathbf{p}_{2}$.

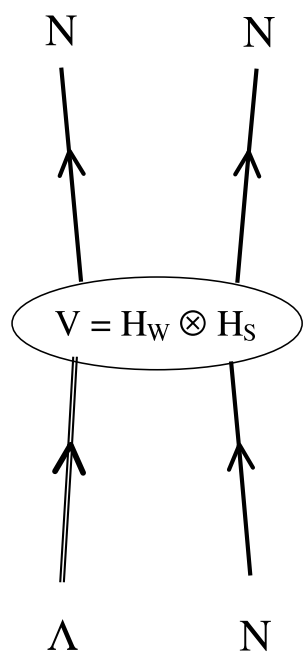

Figure 4. The nonmesonic weak decay mode $\Lambda+N \rightarrow N+N$. In the one meson exchange model a weak vertex $\mathcal{H}_{W}$ is always combined with a strong vertex $\mathcal{H}_{S}$.

We wish to restate that the OMEM transition potential is purely phenomenological and that it is not derived from a fundamental underlying form, as happens for instance, in the case of electro-magnetic transitions or the semileptonic weak decays. As in the case of the OPE, in the OMEM, a weak baryon-baryon-meson (BBM) coupling is always 
combined with a strong BBM coupling. The strong one is determined experimentally with some help from the SU(3) symmetry, and the involving uncertainties have been copiously discussed in the literature [40-43]. It is the weak BBM couplings which could become the largest source of errors. In fact, only the weak $N \Lambda \pi$ amplitude can be taken from the experiment, at the expense of neglecting the off-mass-shell corrections. All other weak BBM couplings are derived theoretically by using $S U(3)$ and $S U(6)_{w}$ symmetries, octet dominance, current algebra, PCAC, pole dominance, etc. [18,24,25,44-51]. Assortments of such methods have been developed and employed for a long time in weak interaction physics to explain the hyperon nonleptonic decays. One should also keep in mind that both the strong and weak BBM couplings, as well the meson masses, can become significantly renormalized by the nuclear environment [51].

The nuclear structure frameworks utilized in the literature for the formal derivations of the NMD rates are: i) the nuclear matter, and ii) the nuclear shell model. There are relatively few works where the second method was employed, and up to quite recently all they were involved the technique of coefficients of fractional parentage, with the spectroscopic factors (SF) explicitly appearing in the expressions for the transition rates [19, 24, 29, 37]. At variance, Barbero et al. [38] have developed a fully general shell model formalism and have specified it for hypernuclei with odd-mass core, such as ${ }_{\Lambda}^{4} \mathrm{H},{ }_{\Lambda}^{4} \mathrm{He},{ }_{\Lambda}^{12} \mathrm{C}$, and ${ }_{\Lambda}^{28} \mathrm{Si}$, were the cores are: ${ }^{3} \mathrm{H},{ }^{3} \mathrm{He},{ }^{11} \mathrm{C}$ and ${ }^{27} \mathrm{Si}$. Here we also discuss the hypernuclei with even-even and odd-odd number of protons and neutrons, namely ${ }_{\Lambda}^{5} \mathrm{He}$ and ${ }_{\Lambda}^{17} \mathrm{O}$, and ${ }_{\Lambda}^{3} \mathrm{H}$, and ${ }_{\Lambda}^{11} \mathrm{~B}$.

\section{Shell Model Formalism}

The shell model framework for the NMD rates has been developed in detail in Ref. [38] and here we will just sketch the main steps, employing the same notation. One starts from Fermi's Golden Rule for the decay rate,

$$
\begin{aligned}
\Gamma & =2 \pi \sum_{S M_{S} J_{F} M_{F} T M_{T}} \int\left|\left\langle\mathbf{p}_{1} \mathbf{p}_{2} S M_{S}, J_{F} M_{F} ; T M_{T}|V| J_{I} M_{I}\right\rangle\right|^{2} \\
& \times \delta\left(\epsilon_{p_{1}}+\epsilon_{p_{2}}+\mathcal{E}_{F}-\mathcal{E}_{I}\right) \frac{d \mathbf{p}_{1}}{(2 \pi)^{3}} \frac{d \mathbf{p}_{2}}{(2 \pi)^{3}}
\end{aligned}
$$

where $V$ is the weak hypernuclear potential, and the wave functions for the kets $\left|\mathbf{p}_{1} \mathbf{p}_{2} S M_{S}, J_{F} M_{F} ; T M_{T}\right\rangle$ and $\left|J_{I} M_{I}\right\rangle$ are assumed to be antisymmetrized and normalized. After performing: 1) the transformation to the relative and center of mass (c.m.) momenta, $\mathbf{p}$ and $\mathbf{P}$, and angular momenta $\mathbf{l}$ and $\mathbf{L}$, and 2) the angular momentum couplings: $\mathbf{l}+\mathbf{L}=\boldsymbol{\lambda}, \boldsymbol{\lambda}+\mathbf{S}=\mathbf{J}$ one obtains:

$$
\begin{aligned}
& \Gamma_{t_{N}}=\frac{16 M_{N}^{3}}{\pi} \hat{J}_{I}^{-2} \sum_{S \lambda l L T J J_{F} \alpha} \int_{0}^{\Delta_{F}^{\alpha}} d \epsilon \sqrt{\epsilon\left(\Delta_{F}^{\alpha}-\epsilon\right)} \\
& \times\left|\sum_{j_{N} j_{\Lambda}} \mathcal{M}\left(p P l L \lambda S J T ; j_{\Lambda} j_{N}, t_{N}\right)\left\langle J_{I}\left\|\left(a_{j_{N} t_{N}}^{\dagger} a_{j_{\Lambda} t_{\Lambda}}^{\dagger}\right)_{J}\right\| J_{F}^{\alpha}\right\rangle\right|^{2},
\end{aligned}
$$

where $\hat{J} \equiv \sqrt{2 J+1}, P=2 \sqrt{M_{N} \epsilon}, p=\sqrt{M_{N}(\Delta-\epsilon)}, \Delta_{F}^{\alpha}=\mathcal{E}_{I}-\mathcal{E}_{F}^{\alpha}-2 M_{N}, t_{\Lambda}=-1 / 2, t_{p}=1 / 2, t_{n}=-1 / 2$, and the label $\alpha$ goes over all final states with the same spin and parity. Thus, that the NMD rates, in principle, depend on both: i) the nuclear structure effects through the two-particle $N \Lambda$ parentage coefficients $\left\langle J_{I}\left\|\left(a_{j_{N} t_{N}}^{\dagger} a_{j_{\Lambda} t_{\Lambda}}^{\dagger}\right)_{J}\right\| J_{F}\right\rangle$, and on the transition potential via the elementary transition amplitudes

$$
\begin{aligned}
\mathcal{M}\left(p P l L \lambda S J T ; j_{\Lambda} j_{N}, t_{N}\right) & =\frac{1}{\sqrt{2}}\left[1-(-)^{l+S+T}\right] \\
& \times\left(p P l L \lambda S J ; T M_{T}=t_{\Lambda}+t_{N}|V| j_{\Lambda} j_{N} J ; t_{\Lambda} t_{N}\right) .
\end{aligned}
$$

Here $(\cdots|V| \cdots)$ is the direct matrix element and the factor in front takes care of the antisymmetrization. To evaluate the nuclear matrix element one has to carry out the $j j-L S$ recoupling and the Moshinsky transformation [52] on the ket $\left.\mid j_{\Lambda} j_{N} J\right)$ :

$$
\left.\left.\mid j_{\Lambda} j_{N} J\right)=\hat{j}_{\Lambda} \hat{j}_{N} \sum_{\lambda^{\prime} S^{\prime} \mathrm{nINL}} \hat{\lambda}^{\prime} \hat{S}^{\prime}\left\{\begin{array}{ccc}
l_{\Lambda} & \frac{1}{2} & j_{\Lambda} \\
l_{N} & \frac{1}{2} & j_{N} \\
\lambda^{\prime} & S^{\prime} & J
\end{array}\right\}\left(\operatorname{nINL} \lambda^{\prime} \mid n_{\Lambda} l_{\Lambda} n_{N} l_{N} \lambda^{\prime}\right) \mid \operatorname{nINL} \lambda^{\prime} S^{\prime} J\right)
$$

where $(\cdots \mid \cdots)$ are the Moshinsky brackets [52], and I and $L$ stand for the quantum numbers of the relative and c.m. orbital angular momenta in the $\Lambda N$ system. The explicit expressions for the transition potentials are given in the Ref. [38]. 
When the hyperon is assumed to be weakly coupled to the $A-1$ core, which implies that the interaction of $\Lambda$ with core nucleons is disregarded, one has that $\left|J_{I}\right\rangle \equiv\left|\left(J_{C} j_{\Lambda}\right) J_{I}\right\rangle$, where $J_{C}$ is the spin of the core, and gets

$$
\left\langle J_{I}\left\|\left(a_{j_{N} t_{N}}^{\dagger} a_{j_{\Lambda} t_{\Lambda}}^{\dagger}\right)_{J}\right\| J_{F}\right\rangle=(-)^{J_{F}+J+J_{I}} \hat{J} \hat{J}_{I}\left\{\begin{array}{ccc}
J_{C} & J_{I} & j_{\Lambda} \\
J & j_{N} & J_{F}
\end{array}\right\}\left\langle J_{C}\left\|a_{j_{N} t_{N}}^{\dagger}\right\| J_{F}\right\rangle .
$$

To evaluate the one-particle spectroscopic amplitudes $\left\langle J_{C}\left\|a_{j_{N} t_{N}}^{\dagger}\right\| J_{F}\right\rangle$ we will use the BCS approximation and, it will be assumed that the even-even, odd-even and odd-odd cores are described, respectively, as zero, one and two quasiparticle states. Correspondingly, the state $\left|J_{C}\right\rangle$ goes into $|B C S\rangle, b_{j_{1} t_{1}}^{\dagger}|B C S\rangle$ and $\left(b_{j_{1} t_{1}}^{\dagger} b_{j_{2} t_{2}}^{\dagger}\right)_{J_{2}}|B C S\rangle$, where $|B C S\rangle$ is the BCS vacuum and $b_{j}^{\dagger}=u_{j} a_{j}^{\dagger}-v_{j} a_{j}$ is the quasiparticle creation operator [38]. In all three cases the NMD rate can be cast in the form:

$$
\Gamma_{t_{N}}=\sum_{j_{N}} \sum_{J=\left|j_{N}-j_{\Lambda}\right|}^{J=j_{N}+j_{\Lambda}} v_{j_{N}}^{2} F_{J}\left(j_{N} t_{N}\right) \mathcal{R}_{J}\left(j_{N} t_{N}\right)
$$

where

$$
\mathcal{R}_{J}\left(j_{N} t_{N}\right)=\frac{16 M_{N}^{3}}{\pi} \int_{0}^{\Delta_{j_{N} t_{N}}} d \epsilon \sqrt{\epsilon\left(\Delta_{j_{N} t_{N}}-\epsilon\right)} \sum_{S l L \lambda T} \mathcal{M}^{2}\left(p \operatorname{PlL} \lambda S J T ; j_{\Lambda} j_{N}, t_{N}\right)
$$

and

$$
\Delta_{j_{N} t_{N}}=M_{\Lambda}-M_{N}+\epsilon_{j_{\Lambda} t_{\Lambda}}+\epsilon_{j_{N} t_{N}}
$$

The geometrical factors $F_{J}\left(j_{N} t_{N}\right)$ are:

1. Even-Even core: $\left|J_{C}\right\rangle \rightarrow|B C S\rangle$,

$$
F_{J}\left(j_{N} t_{N}\right)=\hat{j}_{\Lambda}^{-2} \hat{J}^{2}
$$

2. Odd-Even core: $\left|J_{C}\right\rangle \rightarrow b_{j_{1} t_{1}}^{\dagger}|B C S\rangle$,

$$
F_{J}\left(j_{N} t_{N}\right)=\hat{J}^{2} \sum_{J_{F}=\left|j_{1}-j_{N}\right|}^{j_{1}+j_{N}}\left[1+(-)^{J_{F}} \delta_{j_{1} j_{N}} \delta_{t_{N} t_{1}}\right] \hat{J}_{F}^{2}\left\{\begin{array}{ccc}
j_{1} & j_{N} & J_{F} \\
J & J_{I} & j_{\Lambda}
\end{array}\right\}^{2} .
$$

3. Odd-Odd core: $\left|J_{C}\right\rangle \rightarrow\left(b_{j_{1} t_{1}}^{\dagger} b_{j_{2} t_{2}}^{\dagger}\right)_{J_{2}}|B C S\rangle$,

$$
\begin{aligned}
F_{J}\left(j_{N} t_{N}\right)= & \delta_{t_{N} t_{1}} \hat{J}^{2} \hat{J}_{2}^{2} \sum_{J_{F}=\left|J_{1}-j_{2}\right|}^{J_{1}+j_{2}} \hat{J}_{F}^{2} \sum_{J_{1}=\left|j_{1}-j_{N}\right|}^{j_{1}+j_{N}} \hat{J}_{1}^{2}\left[1+(-)^{J_{1}} \delta_{j_{1} j_{N}}\right] \\
& \left\{\begin{array}{lll}
J_{F} & j_{2} & J_{1} \\
j_{1} & j_{N} & J_{2}
\end{array}\right\}^{2}\left\{\begin{array}{lll}
J_{F} & J_{I} & J \\
j_{\Lambda} & j_{N} & J_{2}
\end{array}\right\}^{2}+\left(j_{1} t_{1}\right) \leftrightarrow\left(j_{2} t_{2}\right)
\end{aligned}
$$

It is clear that in (13) $t_{2}=-t_{1}$.

The nuclear structure manifests itself basically through the factors $F_{J}\left(j_{N} t_{N}\right)$, which are engendered by the Pauli principle. Their values for a few cases are given in Table 1. We would like to stress that the quantum numbers $j_{1} t_{1}$ and $j_{2} t_{2}$ stand for the hyperon partners in the initial state, and that $j_{N}$ runs over all proton and neutron occupied states in the initial nucleus. It is amazing to notice that the the last three equations are valid for any hypernucleus, which could be so light as ${ }_{\Lambda}^{3} H$ or so heavy as ${ }_{\Lambda}^{208} \mathrm{~Pb}$. One should also add that the Eq. (8) contains the same physics as the Eq. (5) in Ref. [24] or the Eq. (30) in Ref. [37], with the advantage that we do not have to deal with spectroscopic factors. Of course, neither the initial and final wave functions are needed. From the results displayed in Table 1 it can be seen that in all three cases the coefficients $F_{J}\left(j_{N} t_{N}\right)$ are of the same order of magnitude, which indicates that the nuclear structure effects in the NMD are of minor importance. 
Table 1. Values of $\left(2 j_{N}+1\right) F_{J}\left(j_{N} t_{N}\right)$. The quantum numbers $\left(j_{1}, t_{1}, J_{I}\right)$ in odd-even core nuclei are: $\left(0 s_{1 / 2},-1 / 2,0\right)$ for ${ }_{\Lambda}^{4} \mathrm{He},\left(0 s_{1 / 2}, 1 / 2,0\right)$ for ${ }_{\Lambda}^{4} H,\left(0 p_{3 / 2},-1 / 2,1\right)$ for ${ }_{\Lambda}^{12} C,\left(0 p_{1 / 2},-1 / 2,1\right)$ for ${ }_{\Lambda}^{16} O$, and $\left(0 d_{5 / 2},-1 / 2,2\right)$ for ${ }_{\Lambda}^{28} S i$. Similarly, $\left(j_{1}, t_{1}, j_{1}, t_{1} ; J_{2} J_{I}\right)$ in odd-odd core nuclei are: $\left(0 s_{1 / 2},-1 / 2,0 s_{1 / 2}, 1 / 2 ; 1,1 / 2\right)$ for ${ }_{\Lambda}^{3} H e$, and $\left(0 p_{3 / 2},-1 / 2,0 p_{3 / 2}, 1 / 2 ; 3,5 / 2\right)$ for ${ }_{\Lambda}^{11} B$.

\begin{tabular}{|rcc|ccccccccc|}
\hline$j_{N}$ & $t_{N}$ & $J$ & ${ }_{\Lambda}^{3} H$ & ${ }_{\Lambda}^{4} \mathrm{He}$ & ${ }_{\Lambda}^{4} H$ & ${ }_{\Lambda}^{5} \mathrm{He}$ & ${ }_{\Lambda}^{11} \mathrm{~B}$ & ${ }_{\Lambda}^{12} \mathrm{C}$ & ${ }_{\Lambda}^{16} \mathrm{O}$ & ${ }_{\Lambda}^{17} \mathrm{O}$ & ${ }_{\Lambda}^{28} \mathrm{Si}$ \\
\hline $0 s_{1 / 2}$ & $\mathrm{n}$ & 0 & $3 / 2$ & 2 & 1 & 1 & 1 & 1 & 1 & 1 & 1 \\
\hline $\mathrm{n}$ & 1 & $1 / 2$ & 0 & 3 & 3 & 3 & 3 & 3 & 3 & 3 \\
\hline $\mathrm{p}$ & 0 & $3 / 2$ & 1 & 2 & 1 & 1 & 1 & 1 & 1 & 1 \\
\hline $\mathrm{p}$ & 1 & $1 / 2$ & 3 & 0 & 3 & 3 & 3 & 3 & 3 & 3 \\
\hline $0 p_{3 / 2}$ & $\mathrm{n}$ & 1 & - & - & - & - & $13 / 2$ & 7 & 6 & 6 & 6 \\
\hline & $\mathrm{n}$ & 2 & - & - & - & - & $11 / 2$ & 5 & 10 & 10 & 10 \\
\hline $\mathrm{p}$ & 1 & - & - & - & - & $13 / 2$ & 6 & 6 & 6 & 6 \\
\hline $\mathrm{p}$ & 2 & - & - & - & - & $11 / 2$ & 10 & 10 & 10 & 10 \\
\hline $0 p_{1 / 2}$ & $\mathrm{n}$ & 0 & - & - & - & - & - & - & 0 & 1 & 1 \\
\hline $\mathrm{n}$ & 1 & - & - & - & - & - & - & 2 & 3 & 3 \\
\hline $\mathrm{p}$ & 0 & - & - & - & - & - & - & 1 & 1 & 1 \\
\hline $\mathrm{p}$ & 1 & - & - & - & - & - & - & 3 & 3 & 3 \\
\hline \hline $1 d_{5 / 2}$ & $\mathrm{n}$ & 2 & - & - & - & - & - & - & - & - & 16 \\
\hline $\mathrm{n}$ & 3 & - & - & - & - & - & - & - & - & 14 \\
\hline $\mathrm{p}$ & 2 & - & - & - & - & - & - & - & - & 15 \\
\hline $\mathrm{p}$ & 3 & - & - & - & - & - & - & - & - & 21 \\
\hline
\end{tabular}

\section{Numerical Results and Discussion}

The numerical values of the parameters necessary to specify the transition potential, were taken from Ref. [24], where, in turn, the strong couplings have been taken from Refs. $[39,40]$ and the weak ones from Ref. [23]. The energy difference $\Delta_{j_{N} t_{N}}$ in (10) is evaluated from the experimental single nucleon and hyperon energies. It is a general belief nowadays that, in any realistic evaluation of the hypernuclear NMD rates, the finite nucleon size (FNS) and the short range correlations (SRC) have to be included simultaneously. Therefore, in the present paper both the FNS and SRC renormalization effects are considered, in the way described in Ref. [38]. Under these circumstances, and because of the relative smallness of pion mass, the transition is dominated by the OPE [24, 38].

The numerical calculations were done in the extreme shell model, which implies that the pairing factors $v_{j_{N}}$ were taken to be equal to one (zero) for the occupied (empty) levels. Thus, from the nuclear structure point of view, the only free parameter is the harmonic oscillator length $b$. We evaluate it from the relation $b=1 / \sqrt{\hbar \omega M_{N}}$, and the oscillator energy was estimated from the relation $\hbar \omega=$ $45 A^{-1 / 3}-25 A^{-2 / 3} \mathrm{MeV}$, which is frequently used for light nuclei.

The calculations for $\Gamma_{N M}$ and $\Gamma_{n / p}$ are confronted the experimental data in Tables 2 and in 3 , respectively. Three different OMEM have been employed for the transition potential. Namely: $(\pi)$ only the pion was taken into the account, $(P S)$ all three pseudoscalar mesons $(\pi+\eta+K)$ were included, and $(P S+V)$ also the vector $\left(\rho+\omega+K^{*}\right)$ mesons are considered. The same remarks are pertinent here as in the study [38] where only ${ }_{\Lambda}^{12} C$ has been analyzed. That is: (1) the simple OPE model accounts for $\Gamma_{N M}$, but it fails badly regarding $\Gamma_{n / p}$, (2) when $\eta$ and $K$ mesons are included, the total transition rate is only slightly modified, while $\Gamma_{n / p}$ change significantly, coming somewhat closer to the measured values, and (3) the results are not drastically modified when all vector mesons are built-in.

\section{Summary and Conclusions}

The shell model formalism for the nonmesonic weak decay of the hypernuclei involves a partial wave expansion of the emitted nucleon waves and preserves naturally the antisymmetrization between the escaping particles and the residual core. The general expression (4) is valid for any nuclear model and it shows that the nonmesonic transition rates should depend, in principle, on both: (i) the weak transition potential, through the elementary transition amplitudes $\mathcal{M}\left(p P l L \lambda S J T ; j_{\Lambda} j_{N}, t_{N}\right)$, and (ii) the nuclear structure, through the two-particle $N \Lambda$ parentage coefficients $\left\langle J_{I}\left\|\left(a_{j_{N} t_{N}}^{\dagger} a_{j_{\Lambda}}^{\dagger}\right)_{J}\right\| J_{F}\right\rangle$. The latter explicitly depend on the initial and final wave functions. Yet, as explained in Ref. [38], and because of: a) the inclusive nature of the nonmesonic decay, and b) the peculiar properties of the coefficients $F_{J}\left(j_{N} t_{N}\right)$, this dependence is washed out. In this way we have arrived at a very simple result for transition rates, given by the Eq. (8), which is valid, not only for the hypernuclei with odd-even core (as shown previously [38]), but also for those which have even-even and odd-odd cores, which has been demonstrated here. 
Table 2. Nonmesonic decay rates $\Gamma_{N M}$ in units of $\Gamma^{0}=2.50 \cdot 10^{-6} \mathrm{eV}$. The symbols $\pi$, PS and V stand, respectively, for the transition potentials activated by the pion, the pseudo-scalar $(\pi+\eta+K)$, and the vector $\left(\rho+\omega+K^{*}\right)$ mesons. Experimental data are shown for comparison.

\begin{tabular}{|c|cccc|}
\hline Hypernucleus & $\pi$ & $P S$ & $P S+V$ & $E X P$ \\
\hline${ }_{\Lambda}^{3} H$ & 0.154 & 0.107 & 0.140 & \\
${ }_{\Lambda}^{4} H e$ & 0.546 & 0.357 & 0.507 & \\
${ }_{\Lambda}^{4} H$ & 0.106 & 0.192 & 0.168 & \\
${ }_{\Lambda}^{5} H e$ & 0.553 & 0.508 & 0.609 & $0.41 \pm 0.14[5]$ \\
${ }_{\Lambda}^{11} B$ & 0.835 & 0.737 & 0.880 & $0.95 \pm 0.13 \pm 0.04[6]$ \\
${ }_{\Lambda}^{12} \mathrm{C}$ & 0.971 & 0.820 & 1.000 & $1.14 \pm 0.2[5]$ \\
& & & & $0.89 \pm 0.15 \pm 0.03[6]$ \\
& & & & $1.14 \pm 0.08[8]$ \\
${ }_{\Lambda}^{16} \mathrm{O}$ & 1.136 & 0.969 & 1.171 & \\
${ }_{\Lambda}^{17} \mathrm{O}$ & 1.178 & 1.028 & 1.226 & \\
${ }_{\Lambda}^{28} \mathrm{Si}$ & 1.314 & 1.100 & 1.322 & $1.30 \pm 0.10[9]$ \\
\hline
\end{tabular}

Table 3. The $n / p$ ratios for several hypernuclei. See the Table 2 caption.

\begin{tabular}{|c|cccc|}
\hline Hypernucleus & $\pi$ & $P S$ & $P S+V$ & $E X P$ \\
\hline${ }_{\Lambda}^{3} H$ & 0.491 & 0.664 & 0.506 & \\
${ }_{\Lambda}^{4} H$ & 2.996 & 24.60 & 10.43 & \\
${ }_{\Lambda}^{4} H e$ & 0.108 & 0.045 & 0.061 & $0.25 \pm 0.13[7]$ \\
${ }_{\Lambda}^{5} H e$ & 0.160 & 0.539 & 0.320 & $0.93 \pm 0.55[5]$ \\
& & & & $1.97 \pm 0.67[6]$ \\
${ }_{\Lambda}^{11} B$ & 0.167 & 0.515 & 0.318 & $1.04_{-0.48}^{+0.59}[5]$ \\
& & & & $2.16 \pm 0.58_{-0.45}^{+0.45}[6]$ \\
${ }_{\Lambda}^{12} C$ & 0.137 & 0.416 & 0.258 & $1.33_{-0.81}^{+1.12}[5]$ \\
& & & & $1.87 \pm 0.59_{-1.00}^{+0.32}[6]$ \\
& & & & $1.17_{-0.08-0.18}^{+0.09+0.20}[10]$ \\
${ }_{\Lambda}^{16} O$ & 0.138 & 0.458 & 0.279 & \\
${ }_{\Lambda}^{17} \mathrm{O}$ & 0.159 & 0.518 & 0.315 & \\
${ }_{\Lambda}^{28} \mathrm{Si}$ & 0.145 & 0.477 & 0.294 & $1.38_{-0.11-0.25}^{+0.13+0.27}[10]$ \\
\hline
\end{tabular}

We reproduce satisfactorily the data for the total transition rates with the OMEM parametrization from the literature [24], but the $n / p$-ratios are not well accounted for. Thus, after having acquired full control of the nuclear structure involved in the process, and after having convinced ourselves that the nuclear structure correlations can not play a crucial role, we firmly believe that the currently used OMEM should be radically changed. Either its parametrization has to be modified or additional degrees of freedom have to be incorporated, such as the factorizable terms [53], the axial-vector-meson exchanges [54], or the correlated the correlated $2 \pi$ from Ref. [37].

The authors acknowledge the support of ANPCyT (Argentina) under grant BID 1201/OC-AR (PICT 03-04296), of CONICET under grant PIP 463, of Fundación Antorchas (Argentina) under grant 13740/01-111, and of Croatian Ministry of Science and Technology under grant \# 119222. D.T. thanks the Abdus Salam ICTP Visiting Scholar programme for travel fares. F.K. is a Fellow of the CONICET Argentina. We would like to thank A.P. Galeão, C. Barbero and E. Bauer for very helpful and illuminating discussions.

\section{References}

[1] Q.N. Usmani, and A.R. Bormer, Phys. Rev. C60, 055215 (1999).

[2] A. Montwill, P. Moriarty, D. H. Davis, T. Pniewski, T. Sobczak, O. Adamović, U. Krecker, G. Coremans-Bertrand, and J. Sacton, Nucl. Phys. A234, 413 (1974).

[3] K.J. Nield, T. Bowen, G. D. Cable, D. A. DeLise, E. W. Jenkins, R. M. Kalbach, R. C. Noggle, and A. E. Pifer Phys. Rev. C13, 1263 (1976).

[4] R. Grace, P. D. Barnes, R. A. Eisenstein, G. B. Franklin, C. Maher, R. Rieder, J. Seydoux, J. Szymanski, W. Wharton, S. Bart, R. E. Chrien, P. Pile, Y. Xu, R. Hackenburg, E. Hungerford, B. Bassalleck, M. Barlett, E. C. Milner, and R. L. Stearns, Phys. Rev. Lett. 55, 1055 (1985). Bo87 J.P. Bocquet, M. Epherre-Rey-Campagnolle, G. Ericsson, T. Johansson, J. Konijn, T. Krogulski, M. Maurel, E. Monnand, J. 
Mougey, H. Nifenecker, P. Perrin, S. Polikanov, C. Ristori, and G. Tibell, Phys. Lett. B192, 312 (1987).

[5] J. J. Szymanski, P. D. Barnes, G. E. Diebold, R. A. Eisenstein, G. B. Franklin, R. Grace, D. W. Hertzog, C. J. Maher, B. P. Quinn, R. Rieder, J. Seydoux, W. R. Wharton, S. Bart, R. E. Chrien, P. Pile, R. Sutter, Y. Xu, R. Hackenburg, E. V. Hungerford, T. Kishimoto, L. G. Tang, B. Bassalleck, and R. L. Stearns, Phys. Rev. C43, 849 (1991).

[6] H. Noumi, S. Ajimura, H. Ejiri, A. Higashi, T. Kishimoto, D. R. Gill, L. Lee, A. Olin, T. Fukuda, and O. Hashimoto, Phys. Rev. C52, 2936 (1995).

[7] V.J.Zeps, Nucl. Phys. A639 261c (1998).

[8] H. Bhang, S. Ajimura, K. Aoki, T. Hasegawa, O. Hashimoto, H. Hotchi, Y. D. Kim, T. Kishimoto, K. Maeda, H. Noumi, Y. Ohta, K. Omata, H. Outa, H. Park, Y. Sato, M. Sekimoto, T. Shibata, T. Takahashi, and M. Youn, Phys. Rev. Lett. 81, 4321 (1998).

[9] H. Park, H. Bhang, M. Youn, O. Hashimoto, K. Maeda, Y. Sato, T. Takahashi, K. Aoki, Y. D. Kim, H. Noumi, K. Omata, H. Outa, M. Sekimoto, T. Shibata, T. Hasegawa, H. Hotchi, Y. Ohta, S. Ajimura, and T. Kishimoto, Phys. Rev. C61, 054004 (2000).

[10] O. Hashimoto, S. Ajimura, K. Aoki, H. Bhang, T. Hasegawa, H. Hotchi, Y. D. Kim, T. Kishimoto, K. Maeda, H. Noumi, Y. Ohta, K. Omata, H. Outa, H. Park, Y. Sato, M. Sekimoto, T. Shibata, T. Takahashi, and M. Youn, Phys. Rev. Lett. 88, 042503 (2002)

[11] J-H. Jun, Phys. Rev. C63, 044012 (2001);

[12] J.B. Adams, Phys. Rev. 156, 1611 (1967).

[13] B. H. J. McKellar, and B. F. Gibson, Phys. Rev. C30, 322 (1984).

[14] K. Takeuchi, H. Takaki, and H. Bandō, Prog. Theor. Phys. 73 (1985) 841

[15] D.P. Heddle and L.S. Kisslinger, Phys. Rev. C33, 608 (1986).

[16] H. Bandõ, T. Motoba, and Žofka, Int. J. Mod. Phys. A21 (1990) 4021

[17] J. Cohen, Prog. Part. Nucl. Phys. 25, 139, edited by A. Faessler, (Pergamon, 1990).

[18] W.M. Alberico, A. De Pace, M. Ericson, and A. Molinari, Phys. Lett. B256, 134 (1991).

[19] A. Ramos, E. van Meijgaard, C. Bennhold, and B.K. Jennings, Nucl. Phys. A644, 703 (1992).

[20] A. Ramos, E. Oset, and L. L. Salcedo, Phys. Rev. C50, 2314 (1995)

[21] A. Parreño, A. Ramos, and E. Oset, Phys. Rev. C51, 2477 (1995)

[22] A. Parreño, A. Ramos, and C. Bennhold, Phys. Rev. C52, R1768 (1995): C54, 1500 (E) (1996)

[23] J. F. Dubach, G. B. Feldman, B. R. Holstein, and L. de la Torre, Ann. Phys. (N.Y.) 249, 146 (1996).

[24] A. Parreño, A. Ramos, and C. Bennhold, Phys. Rev. C56, 339 (1997).

[25] A. Ramos, M.J. Vicente-Vacas, and E. Oset, Phys. Rev. C55, 735 (1997).

[26] E. Oset and A. Ramos, Prog. Part. Nucl. Phys. 41, 191, edited by A. Faessler, (Pergamon, 1998).

[27] A. Parreño, A. Ramos, C. Bennhold, and K. Maltman, Phys. Lett. B435, 1 (1998).
[28] T. Inoue, M. Oka, T. Motoba, and K. Itonaga, Nucl. Phys. A633, 312 (1998).

[29] K. Itonaga, T. Ueda, T. Motoba, Nucl. Phys. A639, 329c (1998).

[30] A. Parreño, A. Ramos, N.G. Kelkar, and C. Bennhold, Phys. Rev. C59, 2122 (1999).

[31] W. M. Alberico and G. Garbarino, Phys. Lett. B486, 362 (2000).

[32] W. M. Alberico, A. De Pace, G. Garbarino, and A. Ramos, Phys. Rev. C61, 044314 (2000).

[33] K. Sasaki, T. Inoue, and M. Oka, Nucl. Phys. A669, 331 (2000), Erratum-ibid A678, 455 (2000).

[34] K. Sasaki, T. Inoue, and M. Oka, Nucl. Phys. A678, 455 (2000).

[35] A. Parreño and A. Ramos, Phys. Rev. C65, 015204 (2001); A. Parreño, A. Ramos, and C. Bennhold, Phys. Rev. C65, 015205 (2001)

[36] E. Oset, D. Jido, and J.E. Palomar, Nucl.Phys. A691, 146 (2001); D. Jido, E. Oset, and J.E. Palomar, Nucl.Phys. A694, 525 (2001).

[37] K. Itonaga, T. Ueda, T. Motoba, Phys. Rev. C65, 034617 (2002).

[38] C. Barbero, D. Horvat, F. Krmpotić, T. T. S. Kuo, Z. Narančić, and D. Tadić, Phys. Rev. C66, 055209 (2002).

[39] M.N. Nagels, T.A. Rijken, and J.J. de Swart, Phys. Rev. D15, 2547 (1977).

[40] P.M.M. Maessen, Th. A. Rijken. and J.J. de Swart, Phys. Rev. C40, 2226 (1989).

[41] D. Halderson, Phys. Rev. C48, 581 (1993).

[42] A. Parreño, A. Ramos, C. Bennhold, and D. Halderson, in Dynamical Features of Nuclei and Finite Fermi Systems, (World Scientific, Singapore, 1994) p. 318.

[43] B. Desplanques, J. Donoghue, and B. R. Holstein, Ann. Phys. (N.Y.) 124, 449 (1980).

[44] R. E. Marshak, Riazuddin, and C.P. Ryan: Theory of Weak Interactions in Particle Physics, (Wiley Interscience, New York, 1969).

[45] L. de la Torre, Ph.D. thesis, University of Masschusetts, 1982.

[46] L.B. Okun: Leptons and Quarks (North Holland, Amsterdam,1982).

[47] E.D. Commins and P.H. Bucksbaum: Weak Interactions of Leptons and Quarks (Cambridge University Press, Cambridge, 1983).

[48] G. Nardulli, Phys. Rev. C38, 832 (1988).

[49] C. Barbero, D. Horvat, F. Krmpotić, Z. Narančić, and D. Tadić, Fizika B10, (2001) 1, and Fizika B10, (2001) 307.

[50] C. Barbero, D. Horvat, F. Krmpotić, Z. Narančić, M.D. Scadron, and D. Tadić, Jour. Phys. G27, (2001) B21.

[51] G.E. Brown and M. Rho, Phys.Rept. 363, 85 (2002).

[52] M. Moshinsky, Nucl. Phys. 13, 104 (1959).

[53] E. Fischbach and D.Tadić Phys. Rep. C6, 125 (1973).

[54] M. Birkel and H. Fritzsch Phys. Rev. D53, 6195 (1996). 